\title{
Embarazo ectópico. Manejo no quirúrgico. Estudio retrospectivo de 4 años
}

\author{
Guido Parra; Ricardo Torrents; Eduardo Denubila*
}

RESUMEN OBJETIVO: Manejo no quirúrgico del embarazo ectópico mediante punción transvaginal (Metotrexate) o conducta expectante.

MATERIAL Y METODOS: Presentamos 80 casos de embarazos ectópicos no roto diagnosticados desde junio de 1989 hasta junio de 1993, entre la $5^{\mathrm{a}}$ y $8^{\mathrm{a}}$ semana, por ecografía transvaginal y HCG cuantitativa positiva y que aceptaron ser manejados en forma no quirúrgica.

RESULTADOS: De los 60 casos que fueron puncionados, 59 se realizaron en forma satisfactoria y sólo una requirió cirugía inmediata por sangrado. No se encontraron efectos secundarios inmediatos por el uso del Metotrexate, la tolerancia fue excelente, comprobándose permeabilidad tubárica por Histerosalpingografía.

De los 20 casos manejados de forma expectante todos mostraron un descenso progresivo de los niveles de HCG excepto un caso que presenta un aumento de los niveles de HCG y requirió microcirugía.

CONCLUSION: La Ecografía transvaginal ha sido de gran ayuda para el diagnóstico y manejo conservador del embarazo ectópico. El tratamiento mediante punción transvaginal con Metotrexate o la conducta expectante han demostrado ser métodos simples y efectivos con buen pronóstico en la fertilidad posterior.

Los criterios completos de inclusión de los pacientes serán expuestos exhaustivamente en el trabajo completo antes del 30 de noviembre.

PALABRAS CLAVES: Embarazo ectópico, manejo no quirúrgico.

SUMMARY OBJECTIVE: Medical management or treatment not surgical to the unrupted ectopic pregnancy though transvaginal puntion with methotrexate or expectant behavior.

MATERIAL AND METHODS: We show 80 ectopic pregnancies unrupted between june 1989 to june 1993 . And between 5 th though 8 th week of gestation diagnosed; with transvaginal ultrasoud and cuantitative positive B-HCG; that accepted to be management with no surgical procedures.

RESULTS: 60 cases were doing with puntion. 59 in a satisfactory manner and just one needed inmediatly surgical procedure for bleeding. There isn't secundary effects with the methotrexate use. Later, the histerosalpingography show not obstruction at the tubes.

Over the 20 cases treatment with expectante observance all the cases showed descendent behavior to the HCG, only one case show increment of the tituls of HCG and needed a microsurgery procedure.

CONCLUSION: Transvaginal ultrasound is a great tool for the diagnoses and conservadore treatment of the ectopic pregnancy. The transvaginal puntion with methotrexate and the expectant procedures than show to be and efective, simple method with a good fertility pronostic.

KEY WORDS: Ectopic pregnancy, medical management.

\section{ABREVIATURAS}

E.E.: Embarazo Ectópico

HCG: Hormona Coriónica Gonadotrófica

E.I. Embarazo Intrauterino

U/L: Unidades/Litro

MTX: Metotrexate

U/S-T.V.: Ultrasonido Transvaginal

HSG: Histerosalpingografía

FIV/ET: Fertilización In Vitro/Transferencia de Embriones

hCS: Hormona de Crecimiento

La incidencia del embarazo ectópico a nivel mundial ha mostrado una tendencia a incrementarse en la última década; cuestión que se ha atribuido (1), al aumento de la enfermedad pélvica inflamatoria, el uso de métodos

Centro de Diagnóstico ultrasonográfico. Clínica La Asunción. Barranquilla. anticonceptivos como el DIU, pero también por otra parte, al uso cada vez mayor de técnicas de reproducción asistida (superovulación, inseminaciones, etc.) y al auge de la cirugía tubárica.

El diagnóstico temprano (antes de su ruptura) del embarazo ectópico (E.E.) especialmente tubárico, se ha visto beneficiado con las técnicas de determinación cuantitativa 
de la hormona Coriónica-Gonadotrófica (H.C.G.) (2), con el desarrollo acelerado de técnicas endosonográficas y últimamente con la aplicación del Doppler-Color, hasta llegar a grados de sensibilidad y especificidad por encima del $90 \%$ (3).

En la última década, muchos autores revivieron algunas técnicas ya publicadas de manejo no quirúrgico del E.E. e innovaron otras como la aplicación de sustancias tóxicas al Trofoblasto (inyectadas por vía laparoscópica o transvaginal endosonográfica) tales como: Metotrexate (4-5), Cloruro Potásico (6), Prostaglandinas (7), Glucosa Hiperosmolar (8), etc. Otros autores han propuesto la posibilidad de tratamientos sistémicos con sustancias como el Metotrexate (9), RU 486 (10), Danazol (11), publicando algunos resultados positivos.

Investigadores en Norteamérica (12) propusieron la posibilidad de adoptar una conducta expectante en el E.E., demostrando la desaparición o reabsorción del tejido trofoblástico y por consiguiente la negativización de los títulos de HCG en sangre, comprobando posteriormente permeabilidad tubárica en la trompa afectada.

Además de mostrar su eficacia en la resolución del E.E., la conducta no quirúrgica preserva en un gran porcentaje la permeabilidad y funcionabilidad tubárica, lo cual haría más elegible a este método como primera opción terapéutica en los pacientes que desearan conservar su fertilidad.

\section{Materiales y métodos}

Durante el período comprendido entre el 10. de junio de 1989 y el 30 de septiembre de 1993, se incluyeron en total 80 pacientes que se repartieron en dos grupos de acuerdo a los siguientes criterios de selección:

A. Conducta expectante:

1. HCG inicial igual o menor a $2.500 \mathrm{U} / \mathrm{L}$ y meseta o caída al tercer día.

2. Diámetro mayor a la visión ecográfica transvaginal igual o menor a dos centímetros.

3. Ausencia de embriocardia.

B. Punción vaginal-Metotrexate Local:

1. HCG igual o menor de $25.000 \mathrm{U} / \mathrm{L}$.

2. Diámetro mayor a la visión ecográfica transvaginal menor o igual a $2 \mathrm{cms}$.

3. La presencia de embriocardia no fue criterio de exclusión.

Todas las pacientes deseaban fertilidad posterior y los E.E. no rotos fueron, las pacientes se encontraban hemodinámicamente estables al inicio del tratamiento y se les informó sobre los beneficios y riesgos de los procedimientos a efectuar, obteniéndose aceptación verbal y compromiso para el cumplimiento de los controles periódicos que los protocolos exigían.

Con los criterios anteriormente expuestos, veinte pacientes se incluyeron en el protocolo de conducta expectante y sesenta en el de punción vaginal metotrexate; se usaron sondas ecográficas transvaginales de alta resolución (ATLUltramark 4, 8 y 9 HDI Doppler-Color) tanto para su diagnóstico como para la punción dirigida. Se realizaron las mediciones de la HCG con un equipo DELFIA Fluorometer, 12-32; por medio de ensayos inmunoflourométricos que reportaron las mediciones en U/L considerando negativas las pruebas de HCG 0-5 U/L y positivos las mayores de 10. Las ecografías fueron realizadas indistintamente por los tres autores y las punciones dirigidas fueron realizadas en su totalidad por uno de ellos (G.P.A.) y la técnica para la misma fue similar a la practicada en aspiración de quistes ováricos y/o en la FVI/ET.

El protocolo de la conducta expectante consistió:

1. Determinación inicial de HCG

2. Determinación de HCG a los 3 días y posteriormente cada semana hasta llegar a la quinta.

3. Diagnóstico ecográfico inicial y de control a la quinta semana.

4. Hemograma inicial y controles clínicos semanales.

El protocolo punción vaginal MTX consistió:

1. Determinación inicial de HCG

2. Determinación semanal de HCG hasta la quinta semana.

3. Medición inicial del diámetro mayor del E.E. y controles ecográficos periódicos de éste y durante el último año de estudio se hizo valoración del comportamiento del flujo mediante el Doppler-Color.

4. Hemograma completo y determinación de pruebas de función hepática inicialmente y luego a la segunda semana postpunción.

La dosis de MTX intraamniótica varió entre 25 y 100 mgs. dependiendo del peso de la paciente y del tamaño del E.E.

La histerosalpingografía (HSG) fue el método usado para la comprobación de la permeabilidad tubárica en trompa afectada.

\section{Resultados}

Las veinte pacientes incluidas en el protocolo de conducta expectante, tuvieron edades entre los 21 y 34 años y su paridad entre 0 y 2 ; de ellas la número 10 (Ver tabla 1) presentó un aumento de la HCG mayor del $50 \%$ del valor inicial a la semana, y la paciente escogió como tratamiento de elección la microcirugía por laparotomía, siendo éste el único caso de este grupo que no siguió el perfil de disminución de la HCG hasta su negativización a la semana quinta; las 19 pacientes restantes presentaron un perfil de HCG con disminución de aproximadamente $30 \%$ de sus valores iniciales al tercer día y un porcentaje de disminución cercano al $100 \%$ a la quinta semana (Ver figura 1), y en promedio, los niveles iniciales del HCG se encontraron alrededor de $1.341 \mathrm{U} / \mathrm{L}$ y a la quinta semana se encontraban en menos de $20 \mathrm{U} / \mathrm{L}$ (Ver figura 2).

De los 19 casos, a 12 se le practicaron HSG confirmándose permeabilidad tubárica en 11 de.ellos, de los cuales 8 presentaron embarazo intrauterino (E.I.) y una presentó E.E. (Ver tabla 2). Si totalizamos entre las pacientes que se realizaron HSG y las que no, encontramos que de los 19 casos que se solucionaron con la conducta expectante hubo un total de 11 E.I., y la única repetición del E.E. se presentó en una paciente a quien se le había realizado HSG que confirmó su permeabilidad tubárica en la trompa inicialmente afectada, (caso 7, ver tabla 1).

En resumen el porcentaje de pacientes embarazadas correspondería a $63 \%$ (E.I. $58 \%$ y E.E. 5\%) y en este grupo 
Tabla 1

REGISTRO DATOS GENERALES CONDUCTA EXPECTANTE

\begin{tabular}{|cccccccccc|}
\hline PACI & AME & ECO & HCG & 2-3 DIA1. & SEM 2. & SEM 3. & SEM 4. & SEM 5. & SEM \\
\hline 1 & 5 & 1 & 1100 & 950 & 750 & 460 & 140 & 0.5 & 0 \\
2 & 6 & 1.3 & 750 & 200 & 100 & 30 & 0.5 & 0 & 0 \\
3 & 6 & 1.4 & 1150 & 800 & 340 & 80 & 0.5 & 0 & 0 \\
4 & 7 & 1.4 & 1750 & 1000 & 650 & 500 & 200 & 120 & 0.5 \\
5 & 6 & 1 & 850 & 500 & 170 & 35 & 20 & 0.5 & 0 \\
6 & 5 & 1 & 900 & 800 & 300 & 100 & 50 & 0.5 & 0 \\
7 & 6 & 1.5 & 730 & 300 & 125 & 20 & 0.5 & 0 & 0 \\
8 & 6 & 1.2 & 1200 & 850 & 500 & 150 & 100 & 20 & 0.5 \\
9 & 6 & 1.1 & 1500 & 1100 & 630 & 250 & 200 & 80 & 0.5 \\
10 & 5 & 1.5 & 1800 & 1800 & $2800 *$ & & & 0 & 0 \\
11 & 5 & 1.4 & 1300 & 930 & 520 & 140 & 50 & 30 & 0.5 \\
12 & 5.5 & 1.5 & 1100 & 810 & 325 & 135 & 45 & 0.5 & 0 \\
13 & 6.3 & 1.4 & 1430 & 920 & 240 & 25 & 0.5 & 0 & 0 \\
14 & 6 & 1.6 & 1950 & 1145 & 480 & 205 & 70 & 45 & 0.5 \\
15 & 6.6 & 1.2 & 1900 & 1205 & 630 & 260 & 110 & 0.5 & 0 \\
16 & 6.5 & 1 & 1350 & 730 & 150 & 55 & 0.5 & 0 & 0 \\
17 & 7 & 1.1 & 1700 & 965 & 290 & 105 & 55 & 30 & 0.5 \\
18 & 5.5 & 1.3 & 980 & 470 & 300 & 245 & 85 & 0.5 & 0 \\
19 & 6.2 & 1.5 & 1630 & 765 & 218 & 150 & 35 & 0.5 & 0 \\
20 & 6.4 & 1 & 1750 & 1140 & 842 & 450 & 90 & 0.5 & 0 \\
\hline SUMA & 119 & 25.4 & 26820 & 17380 & 7560 & 3395 & 1252.5 & 329 & 3 \\
MEDI & 5.95 & 1.27 & 1341 & 869 & 378 & 178.68 & 65.921 & 16.45 & 0.15 \\
$*$ MICROCIRUGIA & & & & & & & & \\
\hline
\end{tabular}

Figura 1

CONDUCTA EXPECTANTE. NIVELES DE HCG

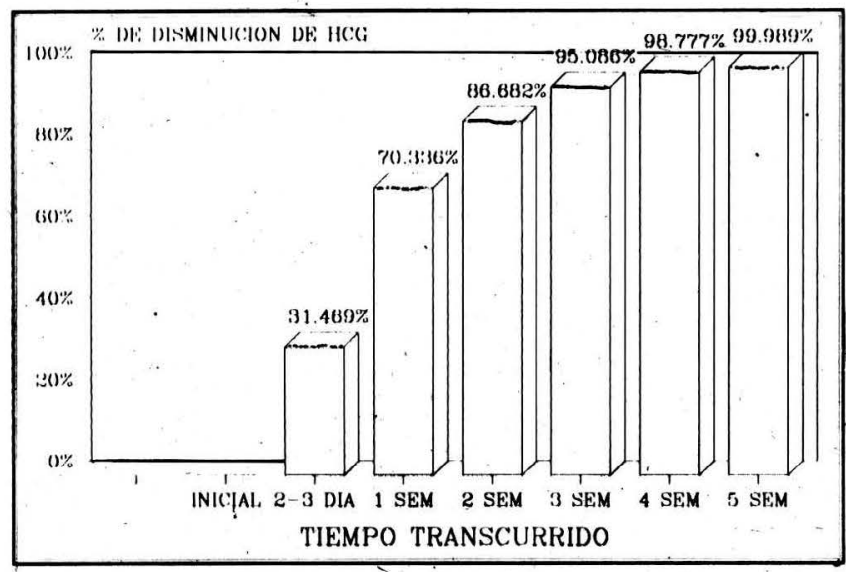

todas las pacientes desearon una búsqueda inmediata del embarazo (Ver figura 3). A los casos incluidos para conducta expectante durante el año de 1993, se les adicionó el criterio del Doppler-Color y el análisis de la onda de flujo, incluyendo aquellas que presentaron escaso flujo peritrofoblástico al Mapeo-Color y ausencia o bajo flujo distólico en su onda fluxométrica.

Los resultados del grupo punción MTX, incluyen 60 pacientes de las cuales, 58 fueron E.E. tubáricos, un embarazo cornual y otro cervical; las dos últimas presentaron resolución del E.E. a la quinta semana con HCG negativa y la paciente del embarazo cornual presentó E.I. posterior-

\section{CONDUCTA EXPECTANTE. NIVELES DE HCG}

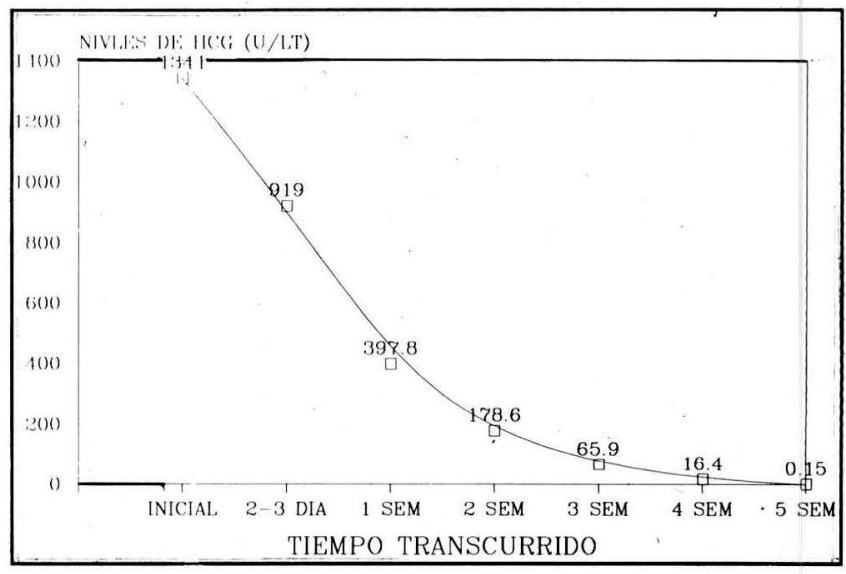

mente (Ver tabla 3, casos 25 y 54). El caso 1, presentó hemoperitoneo postpunción inmediata por lo cual se decidió laparotomía; este caso además de ser el primero en la experiencia presentó el nivel más alto de HCG (25.000 U/ L) y el de mayor tamaño $(3 \mathrm{cms})$ con embriocardia positiva.

Los niveles de $\mathrm{HCG}$ en porcentaje, disminuyeron en un $32 \%$ en la primera semana, lo cual fue significativo al compararlu con el control de la primera semana, de las pacientes de conducta expectante que fue de un $70 \%$ (Ver figuras 1 y 4), pero la tendencia en porcentaje de disminución a la quinta semana fue cercana al $100 \%$. 
Tabla 2

RESULTADOS HSG Y EMBARAZOS (CONDUCTA EXPECTANTE)

\begin{tabular}{|lcl|}
\hline $\mathbf{N}^{\circ}$ Caso & $\begin{array}{l}\text { HSG } \\
\text { 3 Meses }\end{array}$ & Embarazos \\
\hline 2 & + & E.I. \\
3 & + & E.I. \\
6 & + & E.I. \\
7 & + & E.E. \\
8 & + & E.I. \\
9 & + & E.I. \\
11 & - & NO \\
12 & + & E.I. \\
13 & + & NO \\
14 & + & E.I. \\
16 & + & E.I. \\
19 & + & NO \\
\hline TOTAL 12 & $11+$ & 8 (E.I.) \\
& $1-$ & 1 (E.E.) \\
\hline
\end{tabular}

(+) Trompa permeables

(-) Trompa obstruida

E.I. Embarazo intrauterino

E.E. Embarazo ectópico

Figura 3

\section{CONDUCTA EXPECTANTE. EMBARAZOS} POSTERIORES

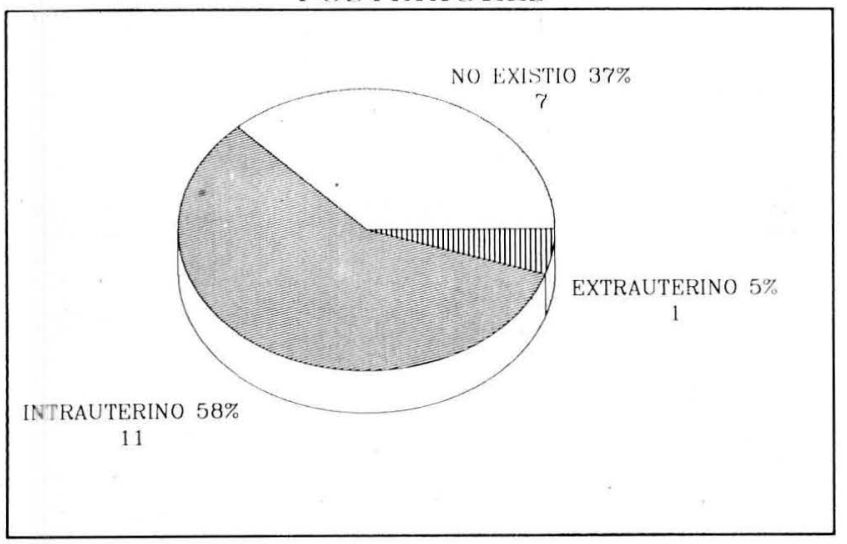

Figura 5

TRATAMIENTO CON PUNCION. NIVELES DE

$\mathrm{HCG}$

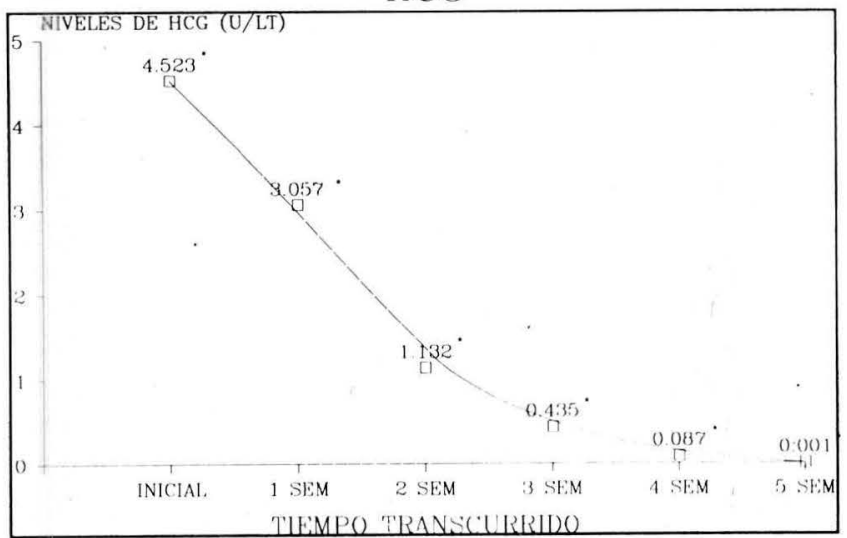

En términos de promedio de HCG, los niveles de ésta estuvieron en $4.523 \mathrm{U} / \mathrm{L}$ en la primera semana y disminuyó a niveles menores de $20 \mathrm{U} / \mathrm{L}$ a la quinta semana (Ver figura 5). Sin embargo, cabe anotar que algunos casos de punción MTX, presentaron un aumento no mayor del $40 \%$ de los valores iniciales, presentando declinación de estos en la segunda determinación de HCG (2da. semana postpunción), por debajo de los valores iniciales (ver figura 5A).

En general, no se observaron efectos secundarios a la aplicación local de MTX, a excepción de ligero dolor en los días siguientes a la punción y sólo un caso presentó leucopenia y dermatosis, que cedieron con la administración de ácido fólico.

El caso 8, a pesar de presentar a la quinta semana HCG negativa, el tamaño de la masa (ectópico) había aumentado de $1.6 \mathrm{cms}$. iniciales a $2 \mathrm{cms}$., y al practicársele HSG se encontró obstrucción tubárica de dicha trompa, por lo que se practicó microcirugía tubárica por laparotomía, y el estudio de anatomía patológica reportó presencia de vellosidades coriales.

De los 60 casos, a 41 se les practicó HSG, mostrando 37 trompas permeables y 4 trompas obstruidas; de las permeables, 22 presentaron E.I. y 1 E.E. (Ver tabla 4). En

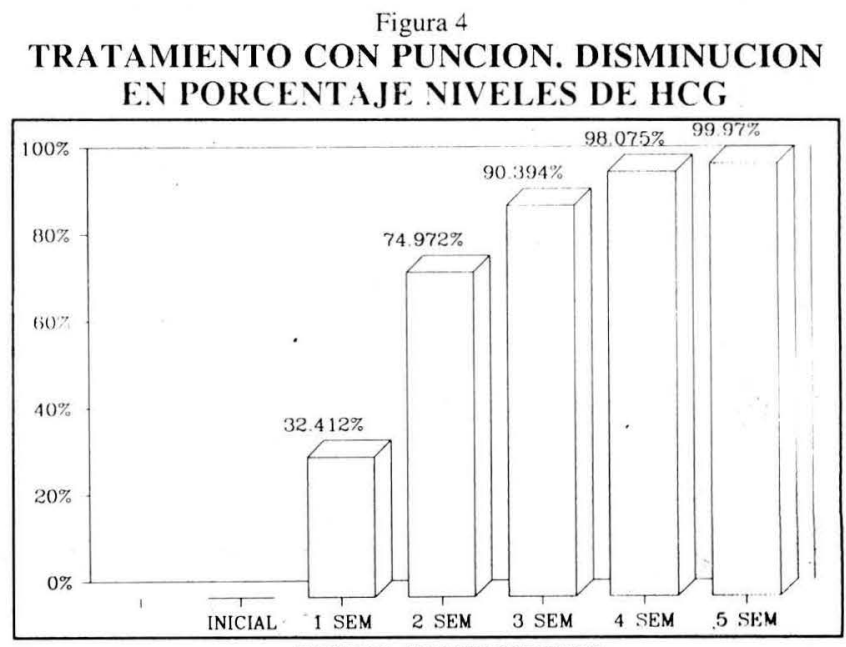

TIEMPO TRANSCURRIDO
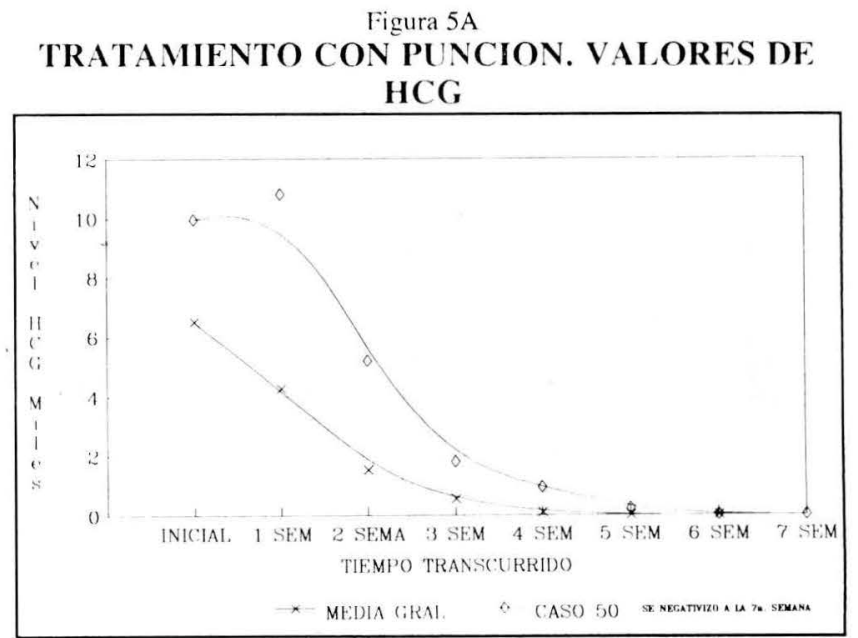
Tabla 3

REGISTRO GENERAL DE CASOS TRATAMIENTO CON PUNCION

\begin{tabular}{|c|c|c|c|c|c|c|c|c|c|c|c|c|c|c|c|}
\hline PACIEN & AME & HCG & TA & EM & 1 & SEMA & 2 & SEMA & 3 & SEM & 4 & SEMA & 5 & \multicolumn{2}{|c|}{ SEMA 3 ME } \\
\hline 1 & 8 & 25000 & 3 & & & & & & & & & & & & \\
\hline 2 & 6 & 2450 & 1.8 & 0 & 1.5 & 2800 & 1 & 1000 & 0 & 450 & 0 & 0.5 & 0 & 0 & 1 \\
\hline 3 & 5 & 3050 & 1.9 & 0 & 1.6 & 1650 & 1.4 & 420 & 1.4 & 70 & 0 & 0.5 & 0 & 0 & 1 \\
\hline 4 & 6 & 4500 & 2 & 0 & 1.3 & 1750 & 0 & 1100 & 0 & 80 & 0 & 0.5 & 0 & 0 & 0 \\
\hline 5 & 7 & 7800 & 2 & 1 & 1.8 & 2400 & 1 & 1600 & 0 & 30 & 0 & 0.5 & 0 & 0 & 1 \\
\hline 6 & 6 & 1500 & 1.2 & 0 & 0 & 240 & 0 & 0.5 & 50 & 0.5 & 0 & 0 & 0 & 0 & 1 \\
\hline 7 & 6 & 890 & 1.4 & 0 & 1.1 & 2400 & 0 & 6 & 0 & 0.5 & 0 & 0 & 0 & 0 & 1 \\
\hline 8 & 6 & 7000 & 1.6 & 1 & 2.2 & 10500 & 2 & 8000 & 2.8 & 1000 & 2 & 80 & 2 & 0.5 & 1 \\
\hline 9 & 5 & 5800 & 1.8 & 0 & 1.5 & 3100 & 1.3 & 1000 & 0 & 320 & 0 & 0.5 & 0 & 0 & 0 \\
\hline 10 & 7 & 14000 & 2 & 1 & 1.3 & 4200 & 1 & 1050 & 0 & 480 & 0 & 0.5 & 0 & 0 & 1 \\
\hline 11 & 7 & 18000 & 2 & 1 & 1.6 & 8000 & 1.5 & 1050 & 1.5 & 850 & 1 & 0.5 & 0 & 0 & 0 \\
\hline 12 & 7 & 7700 & 1.8 & 1 & 1.2 & 6800 & 1.8 & 3700 & 1.5 & 2700 & 1 & 18 & 0 & 0.5 & 0 \\
\hline 13 & 5 & 1350 & 1.1 & 0 & 0 & 260 & 0 & 50 & 0 & 25 & 0 & 0.5 & 0 & 0.5 & 1 \\
\hline 14 & 6 & 2300 & 1.3 & 0 & 1 & 1000 & 1 & 750 & 1 & 300 & 0 & 50 & 0 & 0.5 & 1 \\
\hline 15 & 7 & 7500 & 2 & 0 & 1 & 2300 & 1 & 1000 & 1 & 500 & 0 & 70 & 0 & 0.5 & 1 \\
\hline 16 & 7 & 9500 & 2.5 & 1 & 1.5 & 11000 & 1.3 & 6300 & 1 & 1850 & 0 & 400 & 0 & 20 & 1 \\
\hline 17 & 6 & 1800 & 1.3 & 0 & 1 & 850 & 0 & 200 & 0 & 59 & 0 & 25 & 0 & 0.5 & 1 \\
\hline 18 & 7 & 7100 & 2.3 & 0 & 1.7 & 6300 & 1 & 2400 & 1 & 950 & 1 & 310 & 0.5 & 0.5 & 1 \\
\hline 19 & 7 & 7700 & 2.5 & 0 & 2.3 & 8900 & 1.8 & 3200 & 1.5 & 1950 & 1 & 310 & 0.5 & 0.5 & 1 \\
\hline 20 & 6 & 5800 & 2.1 & 0 & 1.8 & 3400 & 1.9 & 1100 & 1 & 630 & 0.6 & 250 & 0 & 0.5 & 1 \\
\hline 21 & 5 & 3900 & 1.8 & 0 & 1.1 & 1630 & 1 & 1200 & 0.6 & 1150 & 0 & 60 & 0 & 0.5 & 1 \\
\hline 22 & 5 & 3570 & 1.5 & 0 & 1.3 & 3000 & 1 & 650 & 0.8 & 20 & 0 & 0.5 & 0 & 0 & 1 \\
\hline 23 & 5 & 3600 & 1.6 & 0 & 1.3 & 1800 & 1.8 & 320 & 1 & 60 & 0.6 & 0.5 & 0 & 0 & 0 \\
\hline 24 & 6 & 4320 & 1.9 & 0 & 1.5 & 5347 & 1 & 1100 & 0.8 & 340 & 0.3 & 160 & 0.4 & 0.5 & 1 \\
\hline 25 & 6.3 & 8500 & 2.4 & 1 & 2.4 & 5347 & 2 & 550 & 3 & 220 & 1 & 85 & 0.6 & 0.5 & 0 \\
\hline 26 & 7 & 7300 & 2 & 0 & 1.7 & 6380 & 1.3 & 3400 & 1 & 540 & 0.8 & 220 & 0.8 & 0.5 & 1 \\
\hline 27 & 6 & 5320 & 2 & 0 & 2 & 6400 & 1.3 & 1750 & 1 & 635 & 1 & 320 & 1 & 0.5 & 0 \\
\hline 28 & 6 & 6150 & 1.8 & 0 & 1.4 & 5400 & 0.8 & 3300 & 0.3 & 1400 & 0.3 & 650 & 0.3 & 0.5 & 1 \\
\hline 29 & 7 & 9800 & 2.6 & 1 & 2.3 & 7200 & 2 & 1400 & 1.8 & 320 & 1.6 & 90 & 0.3 & 0.5 & 0 \\
\hline 30 & 8 & 13000 & 2.9 & 0 & 2 & 9300 & 1.8 & 3200 & 1.3 & 850 & 0.8 & 130 & 0 & 0.5 & 1 \\
\hline 31 & 5 & 1900 & 1.1 & 0 & 0.8 & 420 & 0.8 & 180 & 0.3 & 60 & 0.3 & 0.5 & 0 & 0 & 0 \\
\hline 32 & 5 & 1960 & 1.2 & 0 & 0.8 & 520 & 0.6 & 340 & 0.3 & 240 & 0.2 & 0.5 & 0 & 0.5 & 1 \\
\hline 33 & 5 & 4300 & 2 & 0 & 1.6 & 3700 & 1 & 0 & 0.8 & 0 & 6 & 0 & 3 & 0.5 & 1 \\
\hline 34 & 5 & 2850 & 1.9 & 0 & 1.6 & 1800 & 0.9 & 750 & 0.3 & 320 & 0.3 & 180 & 0 & 0.5 & 1 \\
\hline 35 & 5 & 2500 & 1.7 & 0 & 1.3 & 1750 & 0.8 & 630 & 0.3 & 320 & 0.3 & 90 & 0 & 0.5 & 0 \\
\hline 36 & 6 & 2480 & 2.8 & 0 & 1.6 & 1500 & 1.2 & 240 & 0.5 & 120 & 0.5 & 0.5 & 0 & 0 & 1 \\
\hline 37 & 7 & 3250 & 1.9 & 0 & 1 & 1150 & 0.5 & 450 & 0.5 & 310 & 0.5 & 33 & 0.3 & 0.5 & 1 \\
\hline 38 & 6 & 3050 & 1.6 & 0 & 1.3 & 3800 & 1 & 950 & 0.8 & 380 & 0.3 & 33 & 0.3 & 0.5 & 1 \\
\hline 39 & 5 & 1870 & 1.5 & 0 & 1 & 320 & 0.3 & 150 & 0.3 & 50 & 0.3 & 0.5 & 0 & 0 & 0 \\
\hline 40 & 5 & 1490 & 1.5 & 0 & 1 & 320 & 0.3 & 120 & 0.8 & 350 & 0.4 & 90 & 0 & 0.5 & 1 \\
\hline 41 & 5 & 1350 & 1.1 & 0 & 1.1 & 1500 & 1 & 320 & 0.8 & 80 & 0 & 0.5 & 0 & 0 & 1 \\
\hline 42 & 6 & 4200 & 1 & 0 & 1 & 3950 & 0.5 & 1100 & 0 & 320 & 0 & 0.5 & 0 & 0 & 1 \\
\hline 43 & 6 & 3500 & 1 & 0 & 1.4 & 2300 & 0.8 & 850 & 0.5 & 315 & 0.5 & 45 & 0.8 & 0.5 & 1 \\
\hline 44 & 8 & 9200 & 2.5 & 0 & 2.3 & 4500 & 1.5 & 1250 & 1.5 & 480 & 1.5 & 120 & 0.8 & 0.5 & 0 \\
\hline 45 & 5 & 1950 & 1 & 0 & 1 & 2400 & 0.8 & 950 & 0.6 & 320 & 0.5 & 0.5 & 0 & 0 & 1 \\
\hline 46 & 5 & 2800 & 1.4 & 0 & 1.1 & 3200 & 0.8 & 1650 & 0.6 & 1000 & 0.6 & 520 & 0.6 & 45 & 0 \\
\hline 47 & 5 & 3300 & 1.9 & 0 & 1.8 & 2800 & 0.9 & 1150 & 0.8 & 930 & 0.8 & 150 & 0.5 & 0.5 & 1 \\
\hline 48 & 6 & 6200 & 2.1 & 1 & 0 & 0 & 2.8 & 540 & 0 & 0 & 0.6 & 0.5 & 0 & 0 & 1 \\
\hline 49 & 6 & 3250 & 1.8 & 0 & 0 & 0 & 0 & 0 & 0 & 320 & 0 & 0.5 & 0 & 0 & 1 \\
\hline 50 & 7 & 9950 & 2.8 & 1 & 2 & 10800 & 1.3 & 5200 & 1.8 & 1800 & 1.5 & 943 & 1 & 220 & 0 \\
\hline 51 & 6 & 3950 & 1.8 & 0 & 0 & 0 & 0 & 0 & 0 & 0 & 0.4 & 120 & 0 & 0.5 & 1 \\
\hline 52 & 5 & 2820 & 1.8 & 0 & 1.1 & 2450 & 0 & 0 & 0 & 0 & 0 & 0 & 0 & 0.5 & 1 \\
\hline 53 & 5 & 1750 & 1.3 & 0 & 1 & 1030 & 0 & 0 & 1.5 & 80 & 0.5 & 0.5 & 0 & 0 & 0 \\
\hline 54 & 7 & 3810 & 2 & 1 & 1.8 & 1100 & 1.1 & 330 & 0.8 & 50 & 0.4 & 0.5 & 0 & 0 & 0 \\
\hline 55 & 7 & 4150 & 1.6 & 0 & 1.4 & 5350 & 1 & 3050 & 0 & 1040 & 0 & 80 & 0 & 0.5 & 1 \\
\hline 56 & 5 & 2200 & 1.8 & 0 & 1.3 & 1350 & 0.4 & 770 & 0.4 & 320 & 0.4 & 150 & 0.4 & 0.5 & 1 \\
\hline 57 & 5 & 2050 & 1.4 & 0 & 1 & 3000 & 1 & 310 & 1 & 415 & 0.8 & 195 & 0.5 & 0.5 & 1 \\
\hline 58 & 5 & 1990 & 1 & 0 & 0.8 & 315 & 0.4 & 48 & 0.4 & 0.5 & 50 & 0 & 0 & 0 & 0 \\
\hline 59 & 5 & 1750 & 0.9 & 0 & 0.7 & 1320 & 0.5 & 490 & 0.5 & 320 & 0.5 & 180 & 0.5 & 0.5 & 1 \\
\hline 60 & 6 & 4330 & 1.6 & 0 & 1.3 & 1910 & 1 & 480 & 0.8 & 150 & 0.6 & 0.5 & 0 & 0 & 0 \\
\hline SUMA & 349.3 & 281350 & 104 & 11 & 76.5 & 194209 & 56.2 & 73095 & 42.2 & 27871 & 31.7 & 6168 & 15.1 & 1301.5 & 41 \\
\hline MEDIA & 5.822 & 4689.2 & 1.74 & 0.183 & 1.275 & 3236.8 & 0.937 & 1218.20 . & .7033 & 464.510. & 0.5283 & 102.8 & 0.25 & 5.0250. & .6833 \\
\hline
\end{tabular}


Tabla 4

\section{RESULTADOS HSG Y EMBARAZOS (PUNCION MTX)}

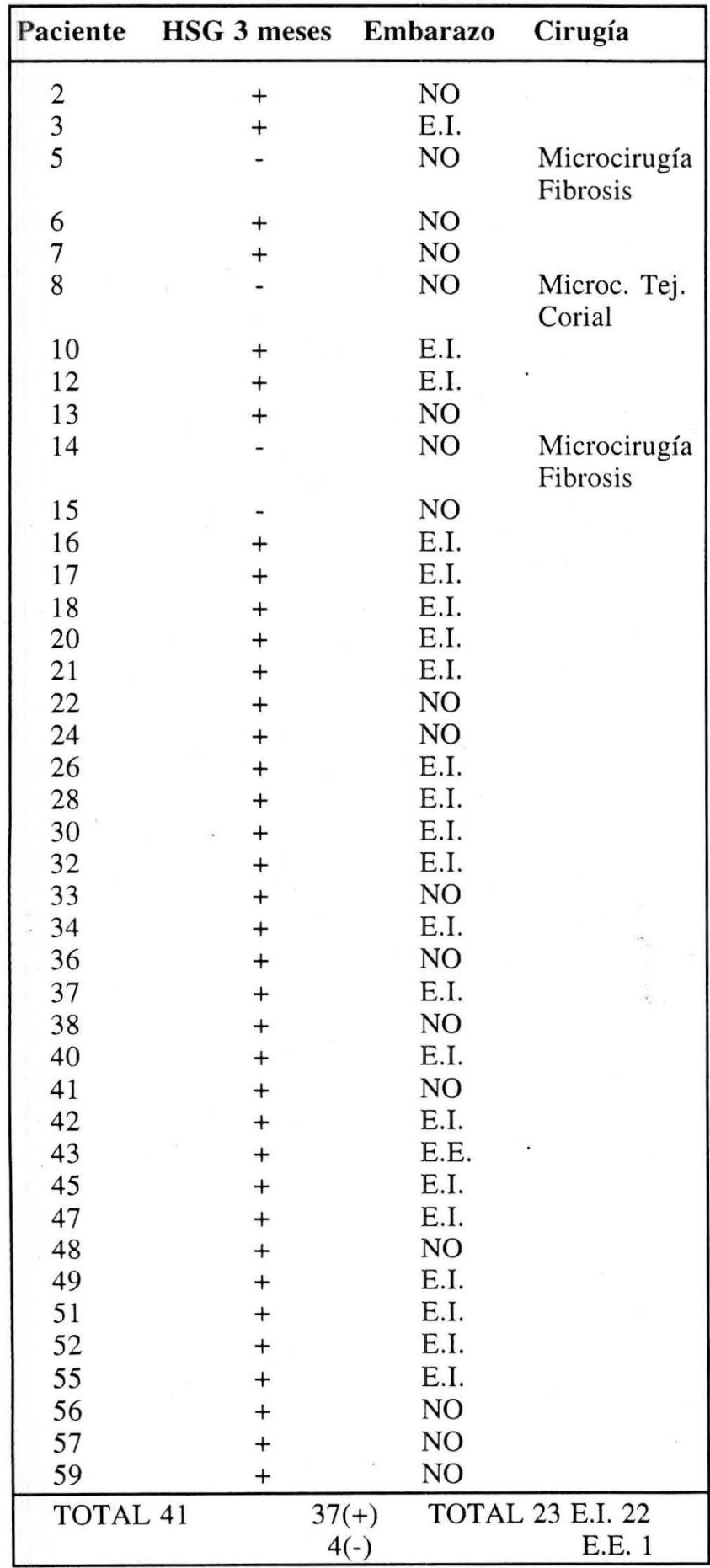

(+) Trompa permeable

(-) Trompa obstruida

E.I. Embarazo intrauterino E.E. Embarazo extrauterino

total, sin tener en cuenta la HSG, 29 se embarazaron, 25 de ellas con E.I. y 4 con E.E. (Ver figura 6). Vale la pena aclarar que del total de pacientes, dos no han deseado embarazo en el período postpunción hasta la fecha de corte del estudio.
De las cuatro pacientes que mostraron trompas obstruidas a la HSG, a dos se les practicó microcirugía tubárica con resección parcial de la misma y reanastomosis, y el informe de patología mencionó fibrosis endoluminal de la trompa en ambos casos.

El estudio comparativo en las pacientes de punción MTX, de aquellos ectópicos que presentaron embrión vivo y las que no presentaron embrión o éste estaba muerto, mostró diferencias significativas en los valores iniciales de

\section{Figura 6 \\ TRATAMIENTO CON PUNCION. EMBARAZOS POSTERIORES}

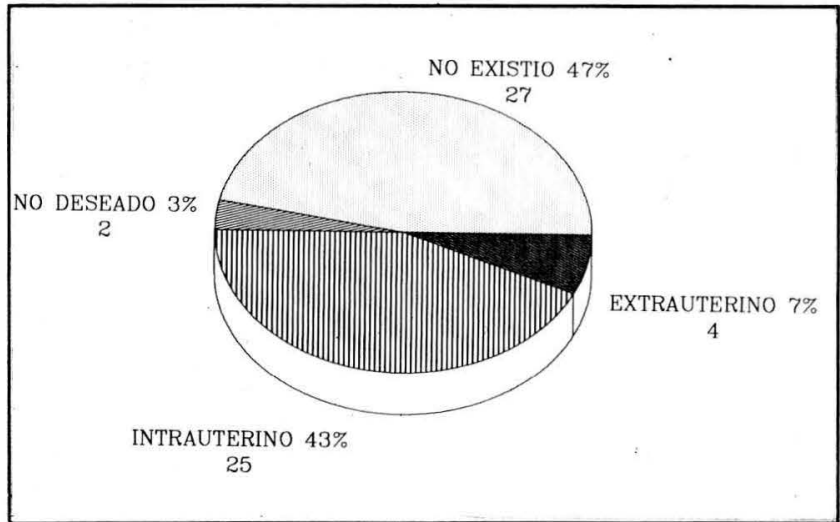

HCG, pero su tendencia es a igualarse alrededor de la cuarta semana, lo cual señalaría un mayor porcentaje de disminución de los valores de HCG en los E.E. con embrión vivo al igual que la reducción del tamaño (Ver figura 7 y 7A). En general, el tamaño del saco gestacional ectópico se relacionó directamente con los valores de HCG y ambos valores declinaron en forma paralela durante la siguiente semana postpunción (Ver figura 8).

Las semanas de amenorrea al momento del diagnóstico variaron entre la quinta y octava semana, y los niveles de HCG se correlacionaron directamente con ellas, presentando una pendiente de disminución más acelerada para los de mayor amenorrea, e igualándose en resultados alrededor de la cuarta y quinta semana (Ver figuras 9 y $9 \mathrm{~A}$ ).

\section{Figura 7 \\ TRATAMIENTO CON PUNCION. VALORES DE HCG}

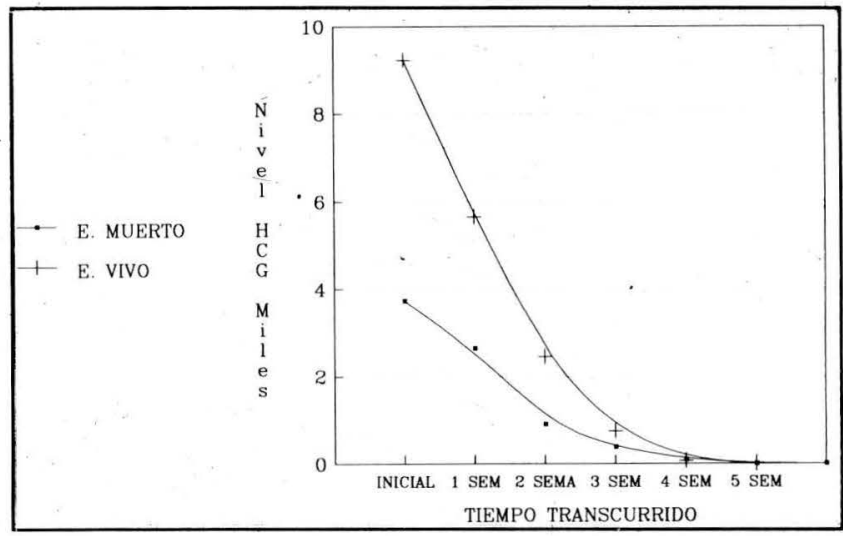


En el último año con el uso del Doppler-Color, observamos que posterior a la punción se presentaba una disminución de la parte diastólica de la onda de flujo y en otros casos hubo una total desaparición de la onda. En la mayoría de los casos, a medida que el tamaño de la masa y los niveles de HCG disminuían, se hacía más aparente la disminución del flujo durante la diástole.

\section{Figura 7A \\ TRATAMIENTO CON PUNCION \\ TAMAÑO SACO}

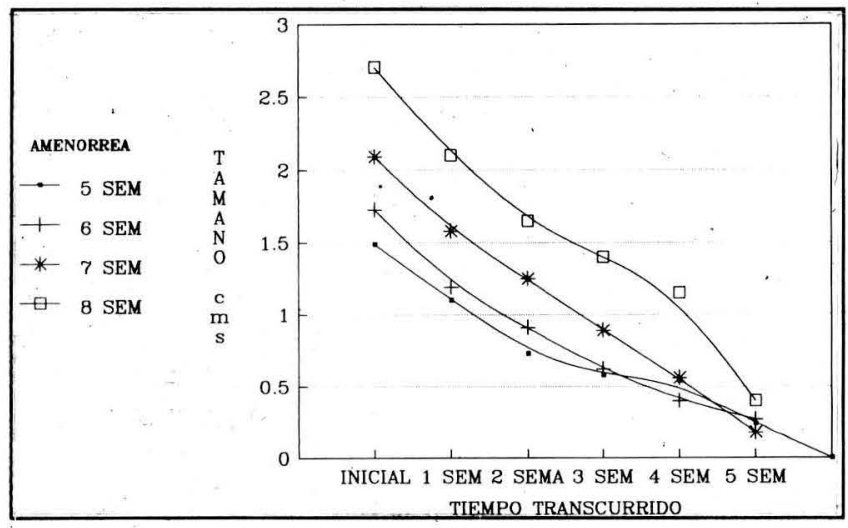

Figura 8

TRATAMIENTO CON PUNCION. RELACION ENTRE TAMAÑO Y NIVELES DE HCG

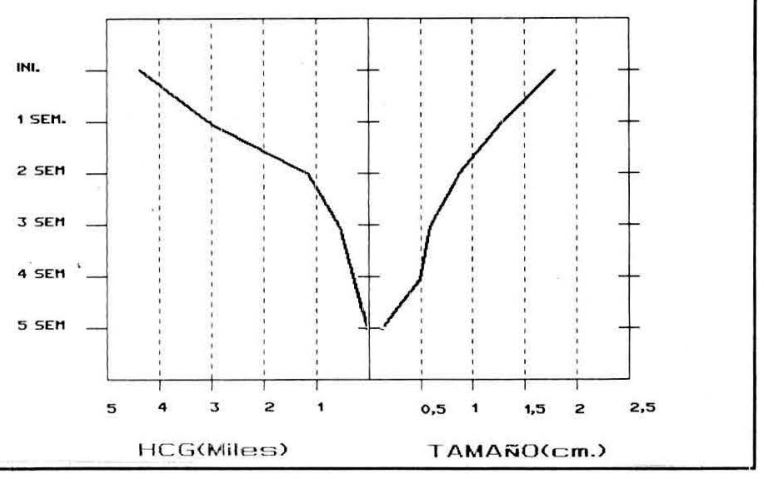

Figura 9

TRATAMIENTO CON PUNCION. VALORES DE HCG

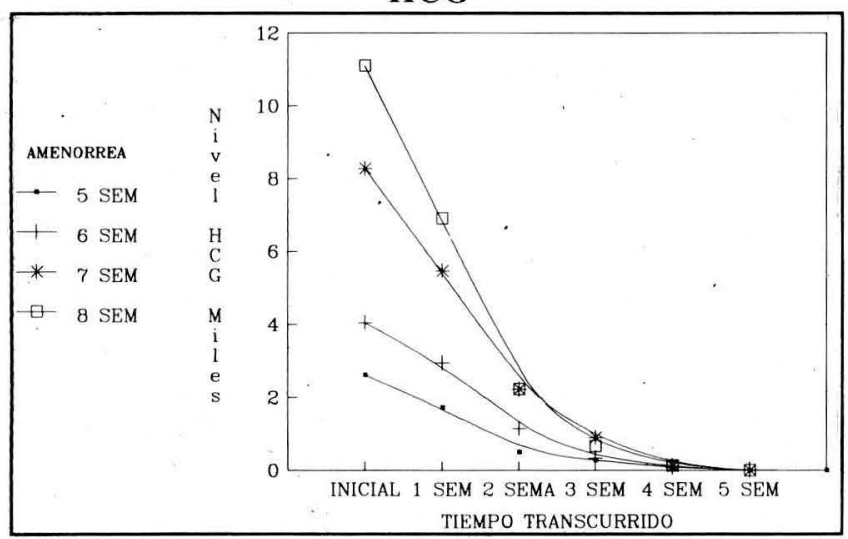

Figura 9A

TRATAMIENTO CON PUNCION. TAMAÑO SACO

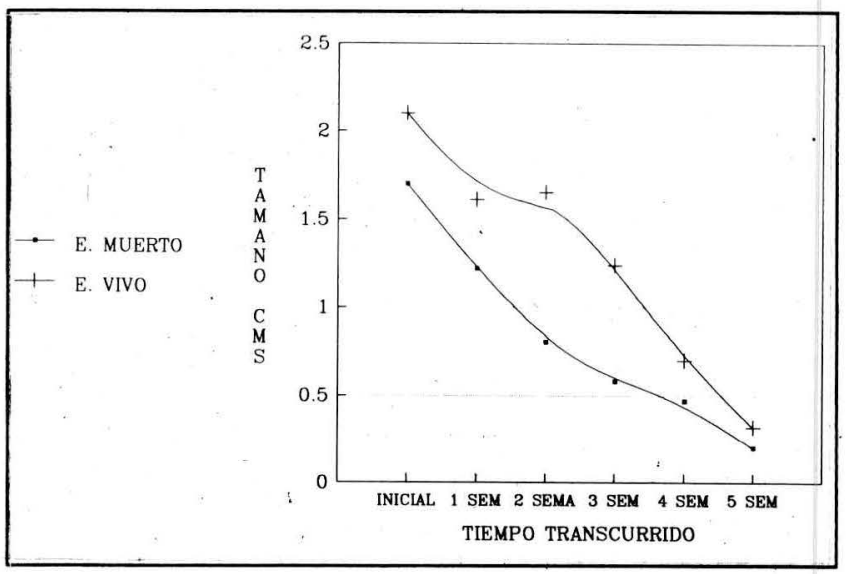

De los casos punción MTX, por escogencia libre, 5 fueron con anestesia general, 45 con anestesia peridural, 8 con local y 2 sin ningún tipo de anestesia. Inicialmente las pacientes fueron hospitalizadas durante las 24 horas siguientes a la punción para observación clínica, y posteriormente a partir de 1991 se hicieron en forma ambulatoria con un tiempo de recuperación que varió entre 1 y 5 horas.

$\mathrm{Si}$ hacemos un corte comparativo con el objeto de valorar si la HCG inicial, podría servir como método predictivo del éxito del resultado de la punción MTX, encontramos que si separamos nuestras pacientes en dos grupos tomando la cifra de $5.000 \mathrm{U} / \mathrm{L}$, (cifra cercana a la media como línea divisoria), los dos casos en los que no hubo resolución se encontraron por encima de $5.000 \mathrm{U} / \mathrm{L}$ y el Chi cuadrado se encontró estadísticamente significativo (Ver tabla 5).

Tabla 5

RESULTADOS VALORES INICIALES HCG

\begin{tabular}{|c|c|c|c|c|}
\hline & Casos & $\%$ & Fallas & $\%$ Falla \\
\hline $\mathrm{HCG}<5000 \mathrm{U} / \mathrm{LT}^{* *}$ & * 39 & $100.00 \%$ & 0 & $0.00 \%$ \\
\hline $\mathrm{HCG}>5000 \mathrm{U} / \mathrm{LT}^{* *}$ & * 19 & $90.48 \%$ & 2 & $9.52 \%$ \\
\hline
\end{tabular}

\section{Discusión}

Nuestra experiencia en conducta expectante muestra una eficacia del 95\% (19 de 20 casos), siendo importante aclarar que ninguna de nuestras pacientes fue hospitalizada para su observación; esta alta eficacia, si la comparamos con el trabajo inicial de Lund en 1995 (13) que fue sorprendentemente alta para esa época (57\%), muestra quizás el gran avance que se ha tenido en las determinaciones cuantitativas de la HCG y el aporte decisivo del ultrasonido transvaginal (U/S-T.V.) en el diagnóstico del E.E. Si bien ellos no reportaron mortalidad materna, su estancia hospitalaria fue hasta de un mes y lógicamente en sus condiciones tuvieron que realizar muchas laparotomías de urgencia. Indudablemente en el transcurso de estos años, varios autores e investigadores han podido seleccionar 
pacientes para conducta expectante, la mayoría de ellos realizando el diagnóstico por laparoscopia (14) y en algunos casos adicionándoles U/S-T.V. (15); casi todos incluyen en su criterio de selección la caída del HCG en las primeras 72 horas del diagnóstico inicial.

García y Cols. (12), en 1987 incluyeron 13 pacientes con estos criterios, con la única diferencia de que el E.E. podría medir igual o menos de $4 \mathrm{cms}$. y reportaron que sólo uno de ellos requirió laparotomía al hacer una meseta la HCG a los 11 días del diagnóstico inicial y además la paciente experimentaba dolor abdominal creciente. La diferencia principal en nuestros resultados, tal vez radique en que no usamos la laparoscopia para hacer el diagnóstico y que todos nuestros casos fueron manejados sin hospitalización.

En 1991, Ylostalo (15) publicó 48 casos de E.E. con manejo expectante, en cuya selección incluyó criterios de U/S-T.V. además de los otros ya mencionados, y practicó cada 2 a 7 días controles de HCG, encontrando que 31 de ellos $(64.6 \%)$ se resolvieron espontáneamente y en los otros $17(35.4 \%)$ se requirió salpingostomía por laparoscopia, debido a que las pacientes experimentaron dolor abdominal y aumento de la HCG. Como dato a resaltar, el autor menciona que el manejo quirúrgico fue más fácil después de 1 ó 2 semanas de manejo expectante que cuando se hizo en la fase aguda.

Publicaciones como la de Stovall (16) comparan exhaustivamente los trabajos de la literatura desde el año 1955 hasta 1991, presentando 285 casos en su conjunto con un porcentaje de éxito en 192 pacientes (67\%) y con E.I. en 44 pacientes $(22.9 \%)$ y en 13 E.E. (6.7\%).

De acuerdo con nuestros resultados el porcentaje de embarazos fue sensiblemente igual $(58 \%)$ a los reportados y probablemente se incrementen a medida que el tiempo transcurra en los casos que fueron tratados durante el último año; solamente en nuestra serie se ha presentado un caso de E.E. que equivaldría a un $5 \%$, a pesar de que no hay diferencias significativas con respecto a lo reportado; pensamos que criterios nuevos de selección, se pudieran adicionar a los índices publicados por otros autores (17), tales como el uso de equipos de ecografía de alta resolución con Doppler-Color o índices fluxométricos, que indicarían el estado hemodinámico real en el área peritrofoblástica (18) del saco gestacional y podrían aumentar más la eficacia de este tratamiento, debido a que la selección se ajustaría al reconocimiento del cual E.E. se está inactivando y va a ser reabsorbido en su totalidad.

Es importante destacar que los casos que se resuelvan con conducta expectante, aparentemente producirían menor daño tubárico que aquellos en los cuales realizamos cualquier tipo de intervención; sin embargo, en nuestra serie no hubo diferencias significativas entre los resultados de permeabilidad tubárica al realizar la HSG $(90.2$ y $91.6 \%)$ respectivamente. Lo anterior podría deberse a la farmacocinética del MTX, que inyectado por vía intratubárica adquiere niveles sanguíneos altos antes de las 2 horas postpunción, pero descienden rápidamente hasta valores indetectables a las 24 horas; mientras que por vía intramuscular sus niveles permanecen elevados por más de 24 horas, en una proporción de 4 a 6 veces mayores que los niveles alcanzados por vía intratubárica (19).
Hasta que un marcador o prueba nos indique con mayor especificidad cuáles E.E. se resolverían espontáneamente y cuáles no, creemos que el uso combinado en el diagnóstico inicial, de HCG cuantitativa y el U/S-T.V. de alta resolución con Doppler-Color, podrían constituir indudablemente dos pilares fundamentales en la decisión de adoptar una conducta expectante y de continuar monitorizando estos casos con HCG periódicas; sin embargo, no podemos olvidar que el uso de otros marcadores como la Hormona del Crecimiento (hCS) (20), podrían indicarnos con mayor sensibilidad cuáles E.E. a pesar de presentar disminución de HCG se romperían.

Para nuestros países en vía de desarrollo, en donde los costos de cualquier tratamiento son determinantes en su escogencia, tendríamos que hacer un estudio comparativo de los gastos generados entre una laparotomía y/o microcirugía tubárica, más la hospitalización, lógicamente en pacientes que deseen preservar su fertilidad, y sumando el valor aproximado de 6 determinaciones de HCG cuantitativa y 2 ecografías transvaginales con o sin DopplerColor, para poder determinar costos-beneficios y determinar así cuál sería entonces el método más adecuado para escoger y difundirlo en nuestro medio.

Al hablar del protocolo punción MTX, indudablemente tenemos que mencionar que el éxito de la terapia medicamentosa del E.E. no roto, se inició con la publicación en la literatura japonesa en el año de 1987 cuando Tanaka y Cols. (21) trataron un E.E. intersticial exitosamente con conservación de la permeabilidad tubárica. Este tratamiento sistémico conllevó al uso de dosis intramusculares cada dos días de MTX a razón de $1 \mathrm{mg} / \mathrm{k}$ de peso y de factor Citrovorum a $0.1 \mathrm{mg} / \mathrm{k}$ en días alternos, hasta que los niveles de HCG cayeran en un $15 \%$ o más en las últimas 48 horas o después de haber administrado la cuarta dosis de MTX; pero los efectos secundarios a dicho tratamiento fueron notorios a nivel del conteo plaquetario y de las pruebas de función hepática $(22,4)$, sin embargo estudios recientes están aplicando una sola dosis intramuscular de $50 \mathrm{mg} / \mathrm{m} 2$ de superficie corporal sin factor Citrovorum (23). Feichtinger y Cols. 1987, (5) y Leeton y Cols. 1988, (24) reportaron que la inyección directa de MTX en el saco gestacional a dosis bajas (menores de $30 \mathrm{mg}$ ) producía menos efectos secundarios, con éxitos que variaron entre el 33 y el $100 \%$.

Otros autores inyectaron Cloruro Potásico (25) y PgE2 (26), variando su porcentaje de éxito entre 0 y $100 \%$. Otros autores reportaron la inyección directa de Glucosa Hiperosmolar (27) en el E.E. con éxitos mayores del $80 \%$ pero con tasas de E.E. persistentes mayores que para los casos tratados con MTX o Salpingostomía.

Una de las razones por las cuales escogimos el MTX en inyección intraamniótica fue por la posibilidad de usar menos dosis de las ya publicadas por vía sistémica, ya que al revisar la literatura la mayoría de los efectos secundarios se presentaron en las pacientes que fueron tratadas por vía endovenosa y con tratamientos prolongados; además por la experiencia en nuestro centro con el manejo de las punciones por vía transvaginal.

El porcentaje de eficacia en términos de resolución en nuestra serie, fue del $96.6 \%$ (58 de 60) considerando fracasos al primer caso al cual se le practicó laparotomía inmediata postpunción debido a la presencia de hemoperitoneo 
y el caso número ocho en el que la $\mathrm{HCG}$ en la quinta semana estaba negativa pero una masa de $2 \mathrm{cms}$. persistió en la trompa afectada y el estudio anatomopatológico reportó tejido corial; demostrando también la eficacia en términos de conservación de la permeabilidad tubárica en $90.2 \%$ de las pacientes que se les practicó HSG y un porcentaje de E.I. de $43.1 \%$ ( 25 de 58 ) y un porcentaje de E.E. del $6.8 \%$ ( 4 de 58), que si lo comparamos con estudios publicados de cirugía conservadora por laparotomía (De Cherney y Kase, 1979) (28): $39 \%$ de E.I. y $8 \%$ de E.E. y con tratamiento conservador por laparoscopia (Kechstein y Cols. 1990) (29): $44 \%$ de E.I. y $19 \%$ de E.E., nos hace pensar que nuestros resultados son alentadores tanto en términos de eficacia y conservación de permeabilidad tubárica como también en términos de consecución de E.I.

Creemos que detalles técnicos de la punción podrían tener implicaciones en los resultados, tales como el hecho de practicar aspiración del contenido líquido del saco gestacional antes de la instilación del MTX; esta reducción inicial del volumen del saco gestacional facilitaría que al inyectar el MTX no se produzca un aumento brusco de la presión y ruptura del saco, o en teoría el aumento de la presión podría colapsar los vasos peritrofoblásticos e impedir la difusión y por consiguiente la actividad antitrofoblástica del MTX.

Los estudios de aplicación del Doppler-Color (30) mostraron cambios inmediatos en el flujo que podrían deberse más a un aumento de la presión, que a la actividad antifólica del MTX contra el trofoblasto; se ha demostrado que dosis bajas aun menores de $25 \mathrm{mg}$. (31), solas o mezcladas con otras sustancias tendrían la misma eficacia y conducirían entonces a inyectar menor volumen ( 1 ó $2 \mathrm{cc}$.) en los sacos gestacionales, produciendo por consiguiente menor presión intramniótica. Aunque no podríamos dejar de mencionar que algunos autores han publicado que las dosis bajas de MTX (12,5 a $25 \mathrm{mg}$.) se han relacionado con mayores índices de fracasos $(4,32)$, por lo que creemos que $25 \mathrm{mg}$. puede ser una dosis mínima tope, utilizándola también para los E.E. de menor tamaño con niveles bajos de HCG. Esta afirmación estaría soportada en nuestros resultados de la tabla 5 que muestran diferencias significativas en pacientes con HCG inicial por encima de $5.000 \mathrm{U} / \mathrm{L}$, que aquellas pacientes que presentaron niveles de HCG inicial por debajo de $5.000 \mathrm{U} / \mathrm{L}$.
Si bien, en nuestra serie no podíamos excluir que alguna de nuestras pacientes incluidas en el protocolo punción MTX, podrían haberse solucionado espontáneamente, la escogencia libre por parte de la paciente de este método y los resultados iniciales alentadores, nos hacen creer que no cometimos error al puncionarlos, y pensamos con base en los resultados, que si bien el puncionar e inyectar MTX intramniótico pudiese conllevar a sobretratamiento para algunos ectópicos, brinda también la seguridad y la sencillez de un método que en algunas ocasiones se pudo practicar aún de manera ambulatoria y sin ningún tipo de anestesia, sin correr riesgos innecesarios ante la posibilidad de una ruptura del E.E., ya que como comentábamos anteriormente, no existe un marcador real en la actualidad que nos permita predecir con alta especificidad cuál de los E.E. iría a resolverse o romperse espontáneamente; aunque consideramos que el advenimiento del Doppler-Color podría en el futuro constituirse como ese "marcador ideal" que actualmente no tenemos $(30,33)$.

Indudablemente los E.E. cornuales y cervicales serían más beneficiados con el tratamiento de punción MTX, debido a que las otras alternativas descritas en la literatura (33), son invasivas, producen morbimortalidad y en muchas ocasiones comprometen el futuro genésico de las pacientes.

\section{Conclusión}

El manejo no quirúrgico del E.E. se constituye en una alternativa importante en el tratamiento de aquellas pacientes que deseen conservar su fertilidad y aún en aquellas que no lo desean. El éxito del tratamiento está estrechamente relacionado con los criterios de selección y seguimiento.

\section{Agradecimientos}

A todos los médicos ginecólogos de la región, por la remisión de sus pacientes, sin lo cual hubiese sido imposible la realización de este trabajo.

A la Dra. Erika Hoyos por su colaboración y transcripción del manuscrito.

\section{BIBLIOGRAFIA}

1. Cost J., Job-Spira N., Fernández H., Papiernik E., Spira A. Risk factors for ectopic pregnancy: a care control study in France, with special fours on infection factors. A.J. Epidemial 1991; 133: 839-849.

2. Kadar N., Devore G., Romero R. Discriminatory HCG zone: Its use in the sonographic evaluation for ectopic pregnancy. Obstet. Gynecol. 1981; 58: 156.

3. Kurjak A., Zalud I., Schulman H. Transvaginal Color-Doppler of trophoblastic flow in questionable adnexa. J. Ultrasound. Med. 1991; 10: 685 .

4. Stovall TG., Ling FW., Gray LA., Carson SA., Buster JE. Methotrexate of unruptured ectopic pregnancy: A report of 100 cases. Obstet. Gynecol. 1991; 77: 749-753.
5. Feichtinger W., Kemeter P. Conservative treatment of ectopic pregnancy by transvaginal aspiration under sonographyc control and Methotrexate injection. Lancet. 1987; 1: 381.

6. Timor-Trisch I., Baxi L., Peisner DB. Transvaginal salpingocentesis: a new technique for treating ectopic pregnancy. Am. J. Obstet. Gynecol. 1989; 160: 154.

7. Fernandez H., Baton C., Lelaidier C., Frydman R. Conservative management of ectopic pregnancy: prospective randomized clinical trial of methotrexate versus prostaglandin sulprostone by combined transvaginal and sistemic administration. Fertil Steril 1991; 55: 746.

8. Lang PF., Weiss PAM., Mayer HO., Hass.JG., Hönigl W. Conservative treatment of ectopic pregnancy with local injection of hiperosmolar 
glucose solution or prostaglandin $\mathrm{F} 2$ alfa: a prospective randomized study. Lancet 1990; 356: 78-81.

9. Stovall TG., Ling FW., Buster JE. Outpatient chemotherapy of unruptured ectopic pregnancy. Fertil Steril 1989; 51: 435.

10. Kenigsbery D., Porte J., Hull M., Spitz IM. Medical treatment of residual ectopic pregnancy: RU 486 and Methotrexate. Fertil Steril 1987; 47: 702.

11. Vilos GA. Danazol as primary therapy of ectopic pregnancy. Abstracts book, World Congress of Gynecologic Endoscopy, AAGL 22nd 1993; 149.

12. Garcia AJ., Jauber JS., Sama J., Josimovich JB. Expectant management of presumed pregnancy. Fertil Steril 1987; 48(3): 395.

13. Lund SS. Early ectopic pregnancy treated non surgically. J. Obstet. and Gynecol. Br. Emp. 1955; 62: 70.

15. Sauer MV., Gorrill MJ., Rodi IA et al. Non surgical management of unruptured insthmic ectopic pregnancy: preliminare experience. Int. J. Fertil 1988; 33(2): 116.

16. Stovall TG., Ling FW. Extrauterine Pregnancy. Clinical diagnostic an management. Mc Graw-Hill, Inc. 1993; 276.

17 Fernández H., Lelaidier C., Thouvenez V., Frydman R. The use of a pretherapeutic predictive score to determine inclusion criterial for the nonsurgical treatment of ectopic pregnancy. Hum. Reprod. 1991; 6: 995-998.

18. Tekay A., Louppila P. Color Doppler Flow as indicator of trophoblastic activity in tubal pregnancy detected by transvaginal ultrasound. Obstet. Gynecol. 1992; 80: 995.

19. Schiff E., Buston M., Tsabari A., Mashiach S., Weiner E. Pharmacokinetic of Methotrexate after local tubal injection for conservative treatment of ectopic pregnancy. Fertil Steril 1992; 57: 688-690.

20. Carson SA., Stovall T., Umnstot $\mathrm{E}$ et al. Rising human chorionic somatomammotropin predicts ectopic pregnancy rupture following methotrexate chemotherapy. Fertil Steril 1989; 51: 593.

21. Tanaka T., Hayashi H., Kutsuzawa T., Fujimoto S. Ichince K. Treatment of interstitial ectopic pregnancy with Methotrexate: report of a successful case. Fertil Steril 1987; 37: 851-852.
22. Sauer MV., Gorrill MJ., Rodi IA. Nonsurgical management of unruptured ectopic pregnancy: An extended clinical trial. Fertil Steril 1987; 48: 752 .

23. Stovall TJ., Ling FW., Gray LA. Single-dose Methotrexate for treatment of ectopic pregnancy. Obstet. Gynecol. 1991; 77: 754.

24. Leeton J., Davison G. Nonsurgical management of unruptured tubar pregnancy with intra-amniotic methotrexate: Preliminary report of two cases. Fertil Steril 1988; 50(1): 167.

25. Timor-Tristch IE., Monteagudo A., Matera C., Veit CR. Sonographic evolution of cornual pregnancies treated without surgery. Obstet. Gynecol. 1992; 79: 1044.

26. Ribic-Pucelj M., Novak-Antolic Z., Urhovec I. Treatment of ectopic pregnancy with prostaglandin E2. Clin. Exp. Obstet. Gynecol. 1989; 16: 106.

27. Laatikainer T., Tuomivauru L., Käär K. Comparision of a local injection of hiperosmolar glucose solution with salpingostomy for the conservative treatment of tubal pregnancy. Fertil Steril 1993; 60: 8084.

28. De Cherney A., Kase N. The conservative management of unruptured ectopic pregnancy. Obstet. Gynecol. 1979; 54: 45!

29. Kegstein J., Hepp S., Schneider V. et al. The contac Nd. YAG Laser: A new technique for conservation of the Falopian tube in unruptured ectopic pregnancy. Br. J. Obstet. Gynecol. 1990; 47: 352.

30. Tekay A., Martikainen H., Heikkinen H., Kivela A., Jouppila P. Disappearance of the trophoblastic blood flow in tubal pregnancy after Methotrexate injection. I. Ultrasound. Med. 1993; 12: 615-618.

31. Abolahar MA., Mansour RT., Serour GI. Transvaginal injection of potassium cholide and Methotrexate for the treatment of tubal pregnancy with livel fetus. Hum. Reprod. 1990; 5: 887

32. Fernandez H., Ziegler DD., Bourget Pet al. The place of methotrexate in the management of interstitial pregnancy. Hum. Reprod. 1991; 6: 302.

33. Nelson RM. Bilateral internal Iliac Artery ligation in cervical pregnancy. Conservation of reproductive function. Am. J. Obstet. Gynecol. 1979; 134: 145. 